\title{
Variaciones de la intensidad en el Sol desde el centro hasta el limbo u oscurecimiento del limbo solar
}

\author{
Center to Limb Variations (CLV) of the Solar Intensity or Limb Darkening
}

\author{
Jenny Marcela Rodríguez Gómez ${ }^{* 1,2 @] ~}$ \\ ${ }^{1}$ The Catholic University of America, Washington, USA. \\ ${ }^{2}$ NASA Goddard Space Flight Center, Greenbel, USA.
}

Recibida en 19 de Noviembre, 2021. Aceptado en 20 de Enero, 2022.

\begin{abstract}
Las variaciones de la intensidad solar del centro al limbo solar son conocidas en Inglés cómo Center to Limb Variations $(C L V)$ u oscurecimiento del limbo solar. Estas permiten caracterizar diferentes capas de la atmósfera solar, específicamente cuándo dichas variaciones se calculan usando diferentes longitudes de onda. Además, estas variaciones permiten obtener información sobre algunas características propias de las imágenes observadas desde telescopios terrestres que observan continuamente el Sol, como su calidad y cómo mejorar está a través de ajustes a los perfiles de variación de la intensidad. También se presenta una breve descripción de cómo las variaciones en la intensidad del centro al limbo pueden ayudar a describir la atmósfera de otras estrellas binarias eclipsantes y aquellas que poseen exoplanetas.
\end{abstract}

Palabras clave: Sol, Fotosfera, Cromosfera, Intensidad, Campo magnético.

Center to Limb Variations (CLV) or limb darkening allow us to characterize different layers of the solar atmosphere. It is possible to use different wavelengths. These variations give information about the quality of the images observed from ground-based observatories that continuously observe the Sun. Also, a brief description of how the Center to Limb Variations can help to describe the stellar atmospheres, specifically in eclipsing binary stars and stars with exoplanets.

Keywords: Sun, Photosphere, Chromosphere, Intensity, Magnetic Field.

\section{Introducción}

El Sol es una estrella que posee una estructura estratificada debido al efecto de la gravedad [1]. Compuesta por un núcleo, una zona radiativa, una convectiva, la fotosfera, cromosfera, región de transición y corona [2] 4] y referencias allí. La energía radiativa, específicamente la radiación electromagnética es emitida en la fotosfera. La fotosfera es la capa visible del Sol, generalmente llamada la superficie del Sol. La Figura 1 muestra las variaciones de temperatura en la atmósfera solar, en la fotosfera se observa un valor de temperatura mínimo, mientras a través de la atmósfera (cromosfera, región de transición y corona) la temperatura aumenta, hasta llegar a su máximo en la corona solar la parte más externa del Sol.

La emisión o brillo en la fotosfera puede ser descrita mediante la ley de Planck o más específicamente la ley de Stefan-Boltzmann, esto permite describir la emisión en términos de la temperatura [5]. Sin embargo, el brillo del disco solar no es constante y decrece desde el centro hasta el limbo (extremos), este efecto es llamado limb darkening u oscurecimiento en el limbo (Figura 2). Es importante destacar que este efecto no ocurre solamente en el Sol, está presente también en otras estrellas.

\footnotetext{
* Correo electrónico: jemfisi@gmail.com
}

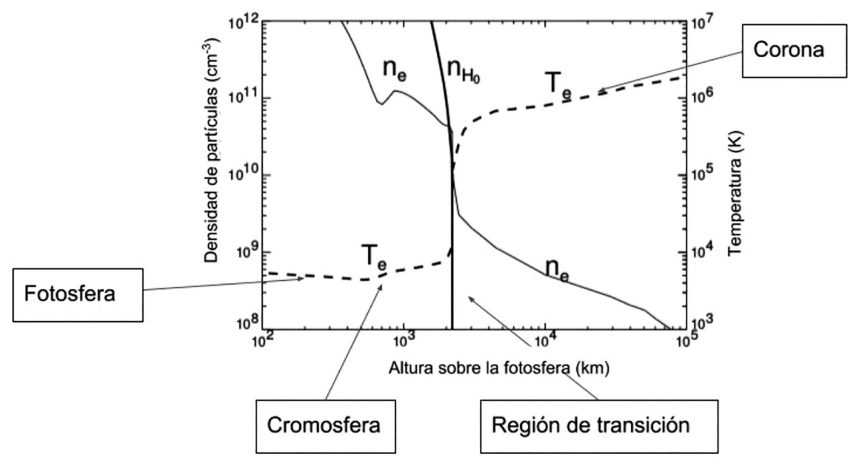

Figura 1: Descripción de las variaciones de temperatura y densidad en la atmósfera solar, la línea punteada muestra la variación de la temperatura. Fuente: [4].

El oscurecimiento en el limbo surge porque cuando observamos directamente al centro del disco solar vemos capas de gas más calientes. Sin embargo, cuando observamos en los extremos o en el limbo estamos viendo capas más altas y frías. Este efecto puede entenderse usando los conceptos de opacidad y profundidad óptica. Podemos observar gas ópticamente espeso (opaco) en el centro, y gas ópticamente delgado (transparente) en el extremo [6]. El oscurecimiento en los extremos surge de la opacidad fotosférica [7]. 


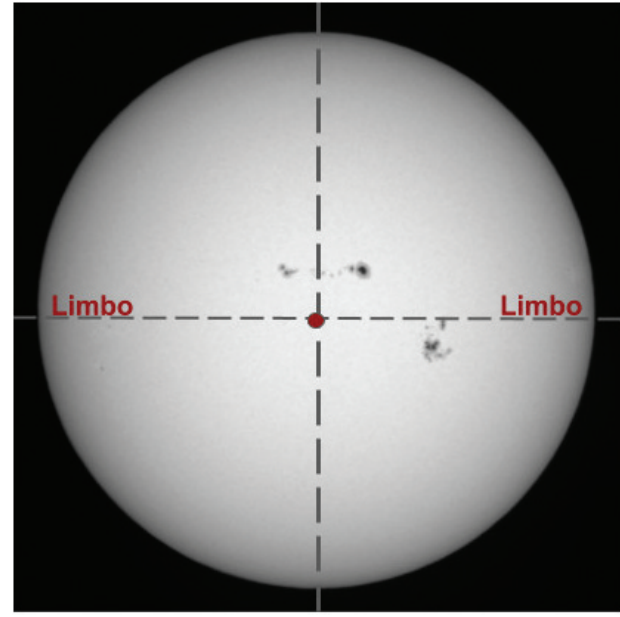

Figura 2: La fotosfera solar observada en luz blanca en 2017-0905 a las 08:15:38 UT, la imagen fue tomada en el observatorio Kanzelhöhe en Austria 1 La imagen muestra el centro del disco solar (punto rojo), la posición del limbo a los extremos del disco solar. Fuente: Autora.

El oscurecimiento en el limbo ha sido ampliamente estudiado, en 1950 Pierce y colaboradores [8, midió el oscurecimiento del limbo solar usando infrarrojo entre 0.5 y $10.2 \mu \mathrm{m}$, encontrando un sistemático incremento en la opacidad solar en longitudes de onda de infrarrojo [9]. Para hacer medidas de la distribución del brillo del disco solar V. Rubin en 1959 [10 describe algunos métodos están disponibles, por ejemplo, la fotografía o fotometría del limbo y el método del eclipse en el cual se analizan fotografías del resplandor solar $u$ observaciones fotoeléctricas obtenidas durante el curso de un eclipse solar total para determinar los coeficientes que caracterizan el oscurecimiento en el limbo [10] y referencias allí.

En 1972 Athay obtuvo perfiles para la variación de la intensidad del centro al limbo en diferentes posiciones $\mu$ usando observaciones de Fe I en el observatorio nacional Kitt Peak 6]. El parámetro $\mu$ corresponde al $\cos \theta$ y $\theta$ es el ángulo entre un rayo emergente y la normal de la superficie solar (Figura 3).

Neckel y Labs en 1994 observaron el oscurecimiento en el limbo entre 1986-1990 usando medidas de telescopios terrestres, pero no encontraron una relación entre el oscurecimiento del limbo y la actividad solar durante el mínimo y máximo del ciclo solar. En 1996 y 2003 Neckel calculó los coeficientes del oscurecimiento del limbo en función de las longitudes de onda y también considerando la temperatura en el limbo [6].

Históricamente, el oscurecimiento del limbo solar ha sido importante en la descripción que la fotosfera se encuentra en equilibrio radiativo, así la energía transportada es producida principalmente por radiación. La convección domina hasta justo debajo de la fotosfera,

\footnotetext{
1 http://cesar.kso.ac.at/
}
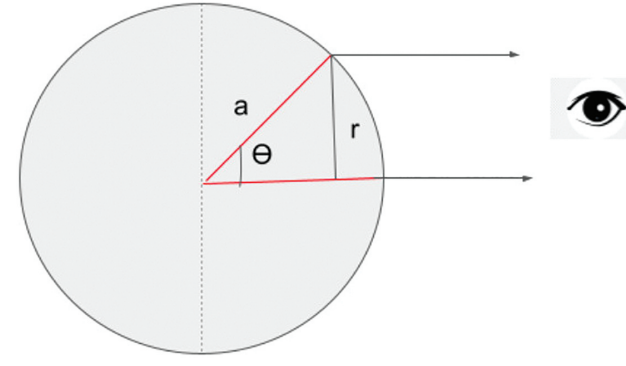

Figura 3: Diagrama del coeficiente del oscurecimiento del limbo, donde $\mu$ corresponde al $\cos \theta$. Fuente autora.

debido al escape de radiación. Además, mostraron la importancia de la extinción de $H^{-}$, pero no generaron modelos muy precisos. En general $H^{-}$es la principal fuente de emisión. También fue posible la determinación de la temperatura con la altura $T(h)$ antes que las intensidades en infrarrojo y ultravioleta estuvieran disponibles para dicho procedimiento [11.

\section{Variaciones de la Intensidad del Centro al Limbo Solar $(C L V)$}

Las variaciones de la intensidad solar del Centro al Limbo o en inglés Center to Limb Variations (CLV), pueden ser descritas usando diferentes expresiones matemáticas, permitiendo extraer información importante de la atmósfera solar. Usualmente estas expresiones permiten obtener una descripción gráfica de las variaciones de intensidad desde el Centro al Limbo solar en tiempos de alta y baja actividad, así como variaciones cuantitativas que permiten describir precisamente las variaciones de la intensidad desde el centro al limbo.

Típicas estimaciones de las variaciones de la intensidad del Centro al Limbo Solar (CLV) pueden ser obtenidas usando la siguiente expresión empírica, para una intensidad específica

$$
I(r)=I(o)\left[1-\mu\left(1-\sqrt{\frac{a^{2}-r^{2}}{a^{2}}}\right)\right]
$$

Donde, $a$ es el radio del disco solar, $r$ es la distancia radial desde el centro del disco, $\mu$ es el coeficiente de oscurecimiento del limbo, $I(o)$ es la intensidad en el centro del disco e $I(r)$ es la intensidad en diferentes posiciones a lo largo del radio 6. Con frecuencia la ecuación 1 puede ser escrita en términos del ángulo $\theta$ [6] (Figura 3)

$$
I(\theta)=I(o)[1-\mu(1-\cos \theta)]
$$

cuando la intensidad del limbo es considerada en el polo, es decir $(r=a)$, la ecuación puede ser escrita como

$$
I(\theta) / I(o)=(1-\mu)
$$

La Ecuación 3 permite obtener el valor del coeficiente de oscurecimiento del limbo para cada perfil, así como un 
(a)

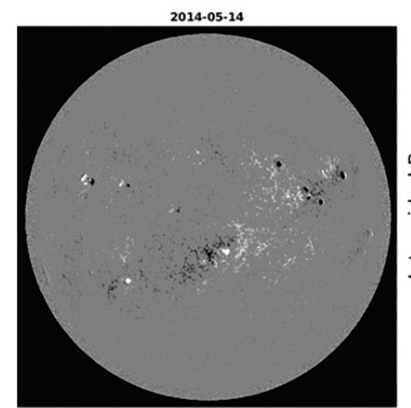

(c)
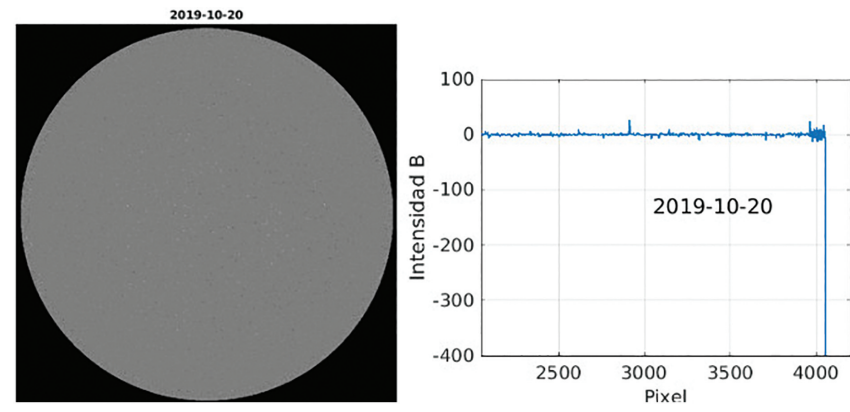

Figura 4: (a) y (c): magnetogramas en un día de mayor actividad solar 2014-05-14 a las 21:48:00 UT y un día de baja actividad o sol calmo en 2019-10-20 a las 21:48:00 UT. (b) y (d) muestra los respectivos perfiles de la intensidad de campo magnético (B) y sus variaciones del Centro al Limbo Solar $(C L V)$, usando la ecuación 3.

estimado de la variación de la intensidad del Centro al Limbo $(C L V)$.

La Figura 4 presenta dos ejemplos de perfiles de variación de la intensidad del Centro al Limbo solar $(C L V)$ en un día de sol calmo (2019-10-20) y un día de gran actividad fotosferica (2014-05-14) usando magnetogramas en la longitud de onda $6173 \mathrm{~A}$, con tamaño $4096 \times 4096$ píxeles, del instrumento $H M I$ abordo de la espacio nave $S D Q^{2}$ (Figura 4).

Los magnetogramas muestran regiones de campo magnético (B) solar con diferentes polaridades positivas (regiones blancas) y negativas (regiones negras) en la Figura 4, así los perfiles de variación del centro al limbo presentan valores positivos y negativos. La variación de la intensidad de campo magnético (B) desde el centro hasta el limbo usando la ecuación 3 permite observar cómo éste refleja periodos de alta actividad solar en la fotosfera caracterizado por manchas solares y regiones activas (panel b Figura 4). De igual manera en periodos de baja actividad o Sol calmo se puede observar como las Variaciones del Centro al Limbo $(C L V)$ muestran variaciones en escalas menores (panel d Figura 4) comparada con las Variaciones del Centro al Limbo cuando regiones activas y manchas solares están presentes.

$\overline{2} \overline{\text { http://jsoc.stanford.edu/ }}$ (a)

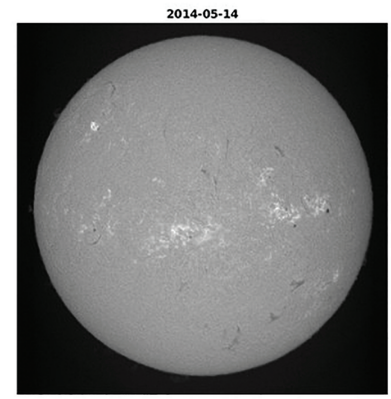

(c)
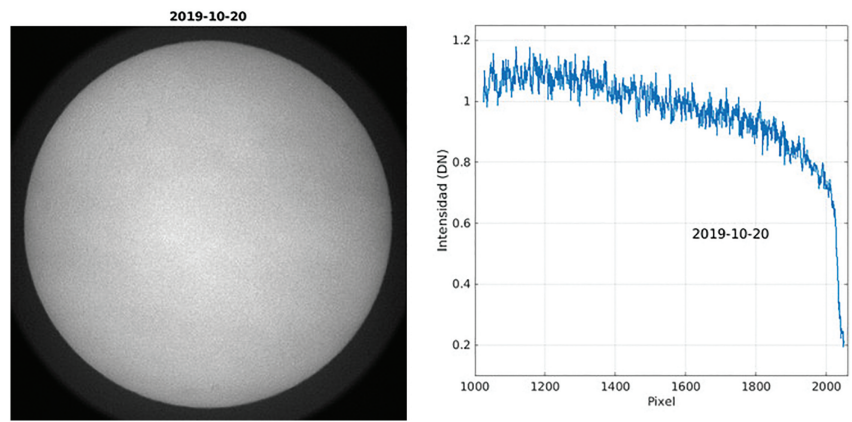

Figura 5: (a) y (c): imágenes en $\mathrm{H}-\alpha$ del observatorio Kanzelhöhe $(\mathrm{KSO})^{3}$ en un día de mayor actividad solar 2014-05-14 a las 09:35:42 UT y un día de baja actividad o Sol calmo en 2019-10-20 a las 07:56:51 UT. (b) y (d) muestra las respectivas variaciones de intensidad del Centro al Limbo Solar (CLV), usando la ecuación 3.

Los perfiles de Variación de la Intensidad del Centro al Limbo $(C L V)$ pueden ser calculados usando imágenes solares que en diferentes longitudes de onda o imágenes como los magnetogramas que miden magnitudes físicas como el campo magnético. Estas imágenes nos permiten obtener información de diferentes capas de la atmósfera solar, por ejemplo, los magnetogramas nos brindan información del campo magnético y fenómenos propios de la fotosfera (la capa visible a simple vista del Sol). Las imágenes en H- $\alpha$ (6562.8 $\AA$ ) proveen información de la cromosfera solar, generalmente esta región se encuentra arriba de la fotosfera brillante y es caracterizada por poseer un gradiente positivo de temperatura. Diferentes eventos toman lugar allí, como prominencias, espículas y fulguraciones entre otras 12 .

Figura 5 muestra variaciones de intensidad del Centro al Limbo $(C L V)$ usando imágenes en $\mathrm{H}-\alpha$ del observatorio Kanzelhöhe (KSO) de la Universidad de Graz, Austria ${ }^{4}$ en 2014-05-14 a las 09:35:42 UT y 2019-10-20 a las 07:56:51 UT. Estas imágenes poseen un tamaño de $2048 \times 2048$ píxeles. Esta figura nos muestra claramente el efecto del oscurecimiento en el limbo solar y más específicamente de la variación de la intensidad del $\sqrt[3]{4 \text { http://cesar.kso.ac.at/ }}$ 
centro del disco solar al limbo $(C L V)$. Al igual que en caso de la intensidad del campo magnético obtenida usando magnetogramas de HMI/SDO (Figura 4), los perfiles de variación de intensidad del Centro al Limbo $(C L V)$ reflejan mayor variación cuando estos se obtienen en tiempos de mayor actividad solar (2014-05-14) en comparación a los obtenidos en tiempos de Sol calmo (2019-10-20) (Figura 5, panel b y c).

\subsection{Variación de la Intensidad del Centro al Limbo $(C L V)$ y su relación con la profundidad óptica}

En general, el flujo desde el disco solar o estelar no es uniforme debido a las profundidades ópticas $(\tau)$, es decir para un específico ángulo de observación, diferentes profundidades del interior solar o estelar pueden ser observadas. La variación de la intensidad del centro al limbo $(C L V)$ proporciona una estimación de la variación de la profundidad óptica $(\tau)$ en diferentes frecuencias. El enfoque clásico de Pierce y Waddell 1961 [13], describe un enfoque de ajuste del oscurecimiento del limbo observado, dependiendo de la frecuencia $\nu$ con los coeficientes $a_{\nu}, b_{\nu}$, and $c_{\nu}$

$$
\frac{I_{\nu}(0, \mu)}{I_{\nu}(0,1)}=a_{\nu}+b_{\nu} \mu+c_{\nu}\left(1-\mu \ln \left(1+\frac{1}{\mu}\right)\right)
$$

donde $I_{\nu}(0,1)$ es la intensidad en el centro, $I_{\nu}(0, \mu)$ es la intensidad del centro al limbo en función de la posición en el disco solar, $\mu$ es el coeficiente de oscurecimiento del limbo. El término exponencial genera un ajuste más preciso que el de un polinomio de tercer orden puede dar [11. Este tipo de descripción nos permite caracterizar las variaciones de la intensidad en diferentes profundidades ópticas o en las diferentes capas solares como la fotosfera, cromosfera, región de transición y corona.

\subsection{Variación de la Intensidad del Centro al Limbo $(C L V)$ y su caracterización usando la regresión no linear de Levenberg-Marquardt}

Pötzi et al. 2021 [14] presentó un método para derivar variaciones de la intensidad del Centro al Limbo $(C L V)$ aplicando un filtro con la mediana en anillos concéntricos en una imagen solar cubriendo el disco solar. Fenómenos como manchas solares, regiones activas o filamentos no tienen influencia en el filtro y son menores que la longitud de cada anillo. El procedimiento es computacionalmente intensivo, debido a que cerca de 900 anillos son procesados. De acuerdo con Scheffler and Elsässer (1990) [15] las variaciones de la intensidad del Centro al Limbo $(C L V)$ pueden ser expresados teóricamente como

$$
\frac{1+\beta \cdot \cos \theta}{1+\beta}
$$

donde $\beta$ es el factor de variación del centro al limbo y $\theta$ es el ángulo de salida de la luz $(\theta=0$ en el centro (a)

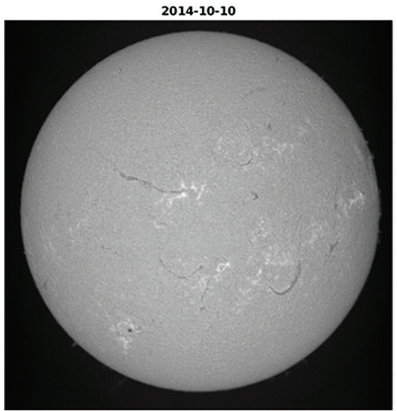

(c)
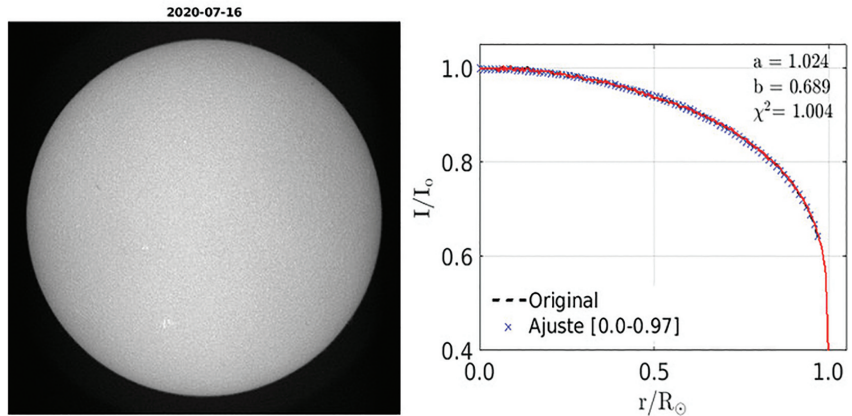

Figura 6: (a) y (c): imágenes en $\mathrm{H}-\alpha$ del observatorio Kanzelhöhe $(\mathrm{KSO})^{5}$ en un día de mayor actividad solar 2014-10-10 a las 08:35:02 UT y un día de baja actividad o Sol calmo en 2020-07-16 a las 10:43:30 UT. (b) y (d) muestra las variaciones de intensidad del Centro al Limbo Solar (CLV) (línea roja), y su respectivo ajuste usando la ecuación 6 (equis azules). $r / R_{\odot}$ corresponde a la distancia desde el centro del disco solar $\left(r / R_{\odot}=0\right)$, hasta el limbo $r / R_{\odot}=1$.

del disco y $\theta=\pi / 2$ en el limbo). Usando las distancias desde el centro del disco solar esta ecuación puede ser reescrita como

$$
y=\frac{(1+b \cdot \cos (\operatorname{asin}(a \cdot x)))}{(1+b)}
$$

donde $a$ es el factor de escala del radio solar, $b$ es el factor de Variación del Centro al Limbo y $x=a \cdot r / R_{\odot}$, donde $R_{\odot}$ corresponde al radio solar. Sin embargo esta expresión no describe el perfil completo de la Variación del Centro al Limbo $(C L V)$ ya que el numerador es siempre más grande o igual que 1 y el límite superior del denominador es definido por $1+b$, en el cual en muchos de los casos es tan pequeño como 2. Para obtener un perfil completo el perfil es expandido computando la mediana. Este procedimiento se ha aplicado a imágenes de en $\mathrm{H}-\alpha$ del observatorio Kanzelhöhe (KSO) de la Universidad de Graz, Austria [14.

La Figura 6 muestra variaciones de intensidad del Centro al Limbo $(C L V)$ usando imágenes en $\mathrm{H}-\alpha$ del observatorio Kanzelhöhe (KSO) de la Universidad de Graz, Austria ${ }^{6}$ para un día con actividad cromosferica

\footnotetext{
5 http://cesar.kso.ac.at/

6 http://cesar.kso.ac.at/
} 
en 2014-10-10 a las 08:35:02 UT y un día de Sol calmo en 2020-07-16 a las 10:43:30 UT (panel a and c). Las variaciones de intensidad del Centro al Limbo (CLV)(línea roja) se observan en el panel (b) y (d), así como su respectivo ajuste usando la ecuación 6 (equis azules), los valores $a$, $b$ y el test Chi-cuadrado $\left(\chi^{2}\right)$ reducido para cada caso, este test permite comparar la bondad de ajuste entre el perfil de variación de la intensidad del Centro al Limbo y su respectivo ajuste usando la ecuación 6 , valores $\geq 1$ muestran un buen ajuste, mientras que valores menores a 1 muestran que el ajuste no es bueno (detalles sobre el test Chi-cuadrado $\left(\chi^{2}\right)$ reducido ver [16]). Pötzi et al. 2021 [14] mostró también el seguimiento durante un día de observación de los perfiles de variación de la intensidad del Centro al Limbo en $\mathrm{H}-\alpha$ y sus ajustes, estos pueden constituir una interesante herramienta para realizar una clasificación de calidad de las imágenes, así como mejorar la calidad de estas.

\section{Aplicaciones en Otras Estrellas}

Estudios de las variaciones de la intensidad del Centro al Limbo solar han sido exploradas en profundidad en el Sol, pero ahora se extienden a otras estrellas con exoplanetas. Durante el tránsito del exoplaneta, porciones de la superficie estelar se ocultan, pero usando la espectroscopía diferencial se pueden obtener espectros de pequeños segmentos de superficie ocultos temporalmente detrás del planeta y calcular las variaciones de intensidad del centro al limbo [17.

Mandel \& Agol (2002) [18] desarrollaron un método analítico comúnmente empleado para ajustar curvas de luz de tránsito, pero esto requiere resolver el radio estelar y la variación del Centro al Limbo $(C L V)$. En general, mientras el planeta pasa frente a la estrella, este bloquea una pequeña fracción de la luz de la estrella, por lo tanto, traza una variación en la intensidad desde el Centro hasta Limbo. Un análisis similar puede ser aplicado para entender curvas de luz de estrellas binarias eclipsantes (Figura 7). De otro lado, observaciones interferométricas comenzaron a mostrar detalles del oscurecimiento del limbo estelar, como el impacto de la convección [19]
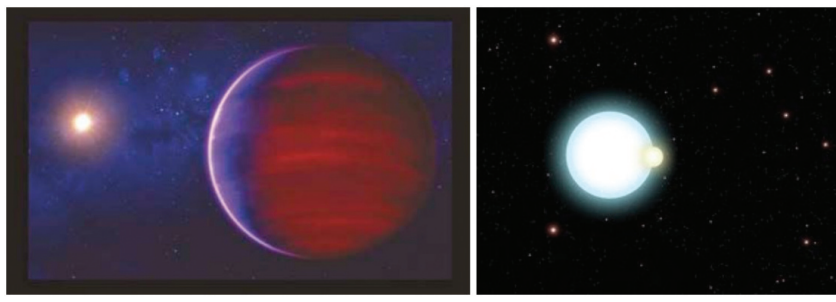

Figura 7: Exoplanetas del tipo Júpiter calientes orbitando otras estrellas (panel izquierdo), estrella eclipsante binaria (panel derecho). Fuente: [23 24]. y la importancia de la extensión en estrellas masivas frías 2022$]$.

La precisión de las observaciones del tránsito planetario está ahora aproximándonos a un punto donde detalles de las atmósferas estelares se vuelven importantes para el análisis de los planetas cercanos a ellas. Así como las curvas de luz de tránsito también pueden ser usadas como modelo para atmósferas estelares [25].

\section{Discusión}

Medir las variaciones de la intensidad del centro al Limbo $(C L V)$ en el Sol, permite describir las diferentes capas de la atmósfera solar y cuantificar variaciones en tiempos de alta actividad solar caracterizada por la presencia de manchas solares, prominencias y fulguraciones entre otras. Así como la baja actividad o Sol calmo donde el disco solar carece de dichas características enunciadas anteriormente (Sección 2, Figuras 4,5 y 6). Así como obtener una evaluación de la calidad de las imágenes haciendo un seguimiento de los parámetros de ajustes de los perfiles de variación de la intensidad del Centro al Limbo. Adicionalmente, mejorar la calidad de las imágenes observadas en observatorios terrestres haciendo uso de los perfiles de variación de la intensidad del Centro al Limbo.

Estas variaciones del Centro al Limbo pueden ser calculadas usando diferentes magnitudes físicas (campo magnético B e intensidad) obtenidas a través del análisis de diferentes longitudes de onda, que a su vez permiten caracterizar la atmósfera solar a diferentes alturas. Diferentes ajustes pueden ser aplicados a estos perfiles de variación del Centro al Limbo, por ejemplo, la regresión no linear de Levenberg-Marquardt y el test Chi-cuadrado $\left(\chi^{2}\right)$ reducido, esto permite caracterizar los perfiles, por ejemplo, en un día de observación, cuantificando sus variaciones, así como relacionar características propias de las observaciones como su calidad.

Las técnicas para determinar las variaciones de la intensidad del centro al limbo constituyen un desafío para otras estrellas, debido a su distancia desde el observador y la dificultad que tenemos de observar diferentes capas de su atmósfera; así como cuantificar variaciones en diferentes magnitudes físicas como la intensidad en diferentes longitudes de onda y su campo magnético entre otras. Sin embargo, la precisión de las observaciones ayudará a obtener información detallada sobre sus atmósferas y permitirá a su vez entender algunas características de los planetas que se encuentran a su alrededor.

\section{Agradecimientos}

Al observatorio Kanzelhöhe (KSO) de la Universidad de Graz, Austria, en especial a Werner Pötzi y a SOLARNET program. 


\section{Referências}

[1] M.J. Aschwanden, Physics of the Solar Corona. An Introduction with Problems and Solutions (Praxis, Chichester, UK; Springer, Berlin, 2005), $2^{\text {a }}$ ed.

[2] J.M. Rodríguez, Revista Brasileira de Ensino de Física 43, e20200495 (2021).

[3] J.M. Rodríguez, F. Carlesso, L.E. Vieira y L. Da Silva, Revista Brasileira de Ensino de Física 40, e03312 (2018).

[4] J.M. Rodríguez, El Sol y la irradiancia. El Sol: conceptos básicos (Editorial Académica Española, 2018).

[5] H.D. Young y A.F. Roger, Física universitaria con física moderna (Pearson Educación, México, 2009), v. 2, $12^{\mathrm{a}}$ ed.

[6] B. Moon, D. Jeong, S. Oh, y J. Sohn, Journal of Astronomy and Space Sciences 34, 99 (2017).

[7] M. Zeilik y S.A. Gregory, Introductory Astronomy and Astrophysics (Cengage Learning, Boston, 1997).

[8] A.K. Pierce, R.R. McMath, L. Goldberg y O.C. Mohler, Astrophys. J. 112, 289 (1950).

[9] A.K. Pierce, C.D. Slaughter y D. Weinberger, Solar Physics 52, 179 (1977).

[10] V.C. Rubin, Astrophys. J. 129, 812 (1959).

[11] J.R. Rutten, Radiative Transfer in Stellar Atmospheres (Sterrekundig Instituut Utrecht, Oslo, 2003).

[12] J.C. Hall, Living Rev. Solar Phys. 5, 2 (2008).

[13] A.K. Pierce y J.H. Memoirs Royal Astron. Soc. 63, 89 (1961).

[14] W. Pötzi, A. Veronig, R. Jarolim, J.M. Rodríguez Gómez, T. Podlachikova, D. Baumgartner, H. Freislich y H. Strutzmann, Solar Physics 296, 164 (2021).

[15] H. Scheffler y H. Elsässer, Physik der Sterne und der Sonne (BI-Wissenschaftsverlag, Mannheim, 1990), $2^{\mathrm{a}}$ ed.

[16] P.R. Bevington y D.K. Robinson, Data reduction and error analysis for the physical sciences (McGraw-Hill, Boston, 2003), $3^{\mathrm{a}}$ ed.

[17] D. Dravins, H. Ludwig, E. Dahlén y H. Pazira, Astronomy \& Astrophysics 605, A91 (2017).

[18] K. Mandel y E. Agol, The Astrophysical Journal 580, L171 (2002).

[19] A. Chiavassa, R. Collet, L. Casagrande y M. Asplund, Astronomy \& Astrophysics 524, A93 (2010).

[20] M. Wittkowski, J.P. Aufdenberg, T. Driebe, V. Roccatagliata, T. Szeifert y B. Wolff Astronomy \& Astrophysics 460, 855 (2006).

[21] M. Wittkowski, J.P. Aufdenberg y P. Kervella, Astronomy \& Astrophysics 413, 711 (2004).

[22] M. Wittkowski, C.A. Hummel, J.P. Aufdenberg y V. Roccatagliata, Astronomy \& Astrophysics 460, 843 (2006).

[23] https://lasp.colorado.edu/outerplanets/exoplanets.php

[24] http://astro.physics.uiowa.edu/ITU/labs/professionallabs/eclipsing-binary-stars/

[25] H.R. Neilson, J.T. McNeil, R. Ignace y J.B. Lester, The Astrophysical Journal 845, 65 (2017). 